\title{
Daily Caffeine Consumption Is Associated with Decreased Incidence of Symptoms and Hemodynamic Changes During Pharmacologic Stress with Regadenoson
}

\author{
Michael I. Posch ${ }^{1}$, Matthew D. Kay ${ }^{1}$, Ahmed A. Harhash ${ }^{2}$, Jennifer J. Huang ${ }^{2}$, Elizabeth A. Krupinski ${ }^{3}$, Aiden Abidov ${ }^{4}$, \\ Natalie A. McMillan ${ }^{1}$, and Phillip H. Kuo ${ }^{1}$ \\ ${ }^{1}$ Department of Medical Imaging, University of Arizona, Tucson, Arizona; ${ }^{2}$ Department of Medicine, University of Arizona, Tucson, \\ Arizona; ${ }^{3}$ Department of Radiology and Imaging Sciences, Emory University, Atlanta, Georgia; and ${ }^{4}$ Department of Medicine, Wayne \\ State University, Detroit, Michigan
}

Regadenoson is an adenosine $\mathrm{A}_{2 \mathrm{~A}}$ receptor agonist widely used as a pharmacologic stress agent for myocardial perfusion imaging. Approximately 3.4 million regadenoson pharmacologic stress tests were performed annually as of 2011. Caffeine is a competitive antagonist of all adenosine receptor subtypes; thus, caffeine is typically withheld $12-24 \mathrm{~h}$ before stress with regadenoson. However, the effects of daily caffeine intake on regadenoson stress are unknown. This study assessed the effects of daily caffeine intake on symptoms and hemodynamic changes during stress testing with regadenoson. Methods: $\mathrm{Pa}-$ tients presenting for regadenoson stress myocardial perfusion imaging were asked their amounts of daily caffeine intake. Chart review was used to collect data on demographics, comorbidities, and use of $\beta$-blockers. Data collected from the regadenoson stress test included symptoms, administration of aminophylline, heart rate, blood pressure, and arrhythmias. $\chi^{2}$ testing and ANOVA were used to analyze data divided into 3 categories of caffeine intake ( $<200,200-400$, and $>400 \mathrm{mg}$ daily). $\chi^{2}$ testing was used for nominal data, and unpaired $t$ testing was used for continuous data. Results: In total, 101 patients were enrolled: $53 \%$ men and $47 \%$ women. Of the 101 patients, $89 \%$ reported caffeine intake, with $13 \%$ reporting heavy caffeine intake $(>400$ $\mathrm{mg}$ daily). The last intake of caffeine was at least $12 \mathrm{~h}$ before the test. During the test, $63 \%$ of patients reported symptoms, but the test was completed successfully in all patients. Compared with those who do not use caffeine, intake for caffeine users was associated with less chest pain $(P=0.0013)$, less aminophylline administration $(P=0.0371)$, lower resting and peak heart rate $(P=0.0497$ and 0.0314 , respectively), and lower diastolic blood pressure response $(P=0.0468)$. No associations were found between caffeine intake and arrhythmia or systolic blood pressure response. Conclusion: The use of regadenoson stress for myocardial perfusion imaging in caffeine consumers is very common, safe, and associated with a lower incidence of certain symptoms than in non-caffeine consumers. Specifically, caffeine intake was associated with less aminophylline use and chest pain.

\footnotetext{
Received Apr. 1, 2019; revision accepted Jul. 23, 2019.

For correspondence or reprints contact: Michael I. Posch, Department of Medical Imaging, University of Arizona, 1501 N. Campbell Ave., Tucson, AZ 85724.

E-mail: michaelianposch@gmail.com

Published online Oct. 11, 2019.

COPYRIGHT (c) 2020 by the Society of Nuclear Medicine and Molecular Imaging.
}

Key Words: regadenoson; caffeine; pharmacologic stress

J Nucl Med Technol 2020; 48:73-76

DOI: 10.2967/jnmt.118.225219

$\mathbf{R}$ egadenoson was approved in 2008 by the U.S. Food and Drug Administration as a pharmacologic stress agent for myocardial perfusion imaging, with approximately 3.4 million regadenoson pharmacologic stress tests performed annually as of 2011 (I). Regadenoson is an adenosine $\mathrm{A}_{2 \mathrm{~A}}$ receptor agonist with a biologic half-life of approximately $1.6 \mathrm{~min}(2)$.

Adenosine nonselectively activates 4 receptor subtypes: $A_{1}, A_{2 A}, A_{2 B}$, and $A_{3}$. Activation of the Gi/o protein-bound $A_{1}$ and $A_{3}$ receptors reduces adenylyl cyclase activity and decreases intracellular cyclic adenosine monophosphate (35). However, activation of the Gs protein-bound $A_{2 A}$ and $\mathrm{A}_{2 \mathrm{~B}}$ receptors increases adenylyl cyclase activity and cyclic adenosine monophosphate levels $(4,5)$. Activation of cardiac $\mathrm{A}_{2 \mathrm{~A}}$ and $\mathrm{A}_{2 \mathrm{~B}}$ adenosine receptors vasodilates the coronary arterial bed, increases myocardial blood flow, and causes sympathoexcitation $(6,7)$.

Caffeine is a competitive antagonist of all adenosine receptor subtypes $(8,9)$. Much of the adult population in the United States consumes caffeine daily, but there is limited literature on the interaction between caffeine and regadenoson. A study by Tejani et al. showed that consumption of caffeine equivalent to 2-4 cups of coffee $90 \mathrm{~min}$ before regadenoson-stress SPECT myocardial perfusion imaging has the potential to adversely affect diagnostic accuracy (10). Previous work by Iskandrian et al. showed that regadenoson is noninferior to adenosine for assessment of myocardial ischemia in patients who have abstained from caffeine for at least $12 \mathrm{~h}(11)$. Both studies focused on the effect of caffeine on diagnostic accuracy rather than patient symptoms and hemodynamic changes, and neither directly evaluated the impact of daily caffeine use. Our study assessed differences in symptoms and hemodynamic changes during stress testing with regadenoson in daily caffeine consumers compared with non-caffeine consumers. 


\section{MATERIALS AND METHODS}

This study was approved by the institutional review board, and the need for written informed consent was waived. Consecutive patients undergoing pharmacologic stress testing with regadenoson between August 2015 and August 2016 were administered a verbal questionnaire prospectively by the nuclear medicine technologist about whether they consume caffeine, their average caffeine consumption per 24-h period, the type of caffeine consumed, and the number of hours since their most recent caffeinated beverage. Patients were asked about daily caffeine intake after the stress test to avoid recall bias. Per institutional protocol, the most recent intake of caffeine was at least $12 \mathrm{~h}$ before the test, and all patients were screened for use of other xanthine derivatives, which were withheld if necessary. Average daily caffeine intake was calculated as follows: 1 cup of coffee was considered equivalent to $100 \mathrm{mg}$ of caffeine; 1 can of soda, to $40 \mathrm{mg}$ of caffeine; and 1 cup of tea, to $25 \mathrm{mg}$ of caffeine. All patients who completed both pharmacologic stress and stress imaging were included, for a total of 101 patients.

Charts were reviewed to obtain data on demographics, diabetes, family or personal history of coronary artery disease, hypertension, hyperlipidemia, smoking, and $\beta$-blocker use. Data collected from the stress test with regadenoson included symptoms, aminophylline use (aminophylline is a regadenoson reversal agent usually administered for severe symptomatic adverse reactions, including nausea, shortness of breath, and headache), physiologic parameters (heart rate, blood pressure, and presence of arrhythmia), electrocardiographic parameters (PR interval, QRS interval, and QTc interval), and SPECT imaging data (left ventricular enlargement and ejection fraction at rest and stress).

Caffeine intake (yes/no) and its relationship to the various variables were analyzed using a $\chi^{2}$ test for nominal data and an unpaired $t$ test for continuous data. When there was a significant result, the data were further analyzed as a function of daily intake $(<200 \mathrm{mg}, 200-400 \mathrm{mg}$, or $>400 \mathrm{mg})$ using $\chi^{2}$ tests for nominal data and ANOVA for continuous data.

\section{RESULTS}

In total, 101 patients were enrolled; 90 were caffeine consumers and 11 were not. Among caffeine consumers, 84 of $90(93.3 \%)$ exclusively consumed regular (nondecaffeinated) coffee, 2 of $90(2.22 \%)$ exclusively consumed decaffeinated coffee, 1 of $90(1.11 \%)$ exclusively consumed soda, and the remaining $3(3.33 \%)$ consumed a mix of coffee, tea, and soda. The sex distribution was $57 \%$ male and $43 \%$ female among caffeine consumers versus $27 \%$ male and $73 \%$ female among non-caffeine consumers $(P=0.0650$, Table 1$)$. Caffeine consumers had a lower average body mass index than noncaffeine consumers (29.40 vs. $33.69, P=0.0439)$. Personal history of coronary artery disease did not differ significantly. The mean age of caffeine consumers was $63 \mathrm{y}$, whereas the mean age of non-caffeine consumers was 58 y $(P=0.1970)$. There was no significant difference between caffeine consumers and non-caffeine consumers in prevalence of diabetes, $\beta$-blocker use, coronary artery disease, hypertension, hyperlipidemia, or tobacco use. There were no significant differences in mean body weight between the 2 groups.

At our institution, aminophylline is used at the discretion of the nurse and only if the patient demonstrates an adverse reaction for longer than $3 \mathrm{~min}$. If the patient does not return to baseline or near baseline, also for longer than $3 \mathrm{~min}$, then aminophylline is also administered. The nursing staff was unaware of the survey; thus, aminophylline administration was not biased by the nurses' knowledge of the patient's daily caffeine intake. Caffeine consumers were significantly less likely to require aminophylline administration than were non-caffeine consumers ( $18 \%$ vs. $45 \%, P=0.0013$; Table 2). No significant difference between caffeine consumers and non-caffeine consumers existed for the following symptoms: palpitations, dyspnea, flushing, headache, nausea, dizziness, feeling hot, dysgeusia, abdominal pain, and back pain. However, caffeine consumers were significantly less likely than non-caffeine consumers to experience chest pain ( $10 \%$ vs. $45 \%, P=0.0371)$.

Caffeine consumers had a significantly lower mean heart rate than non-caffeine consumers at both rest and peak stress (69 vs. $77 \mathrm{bpm}$ at rest, $P=0.0497$, and 97 vs. $108 \mathrm{bpm}$ at peak stress, $P=0.0314$ ), although consumers and nonconsumers experienced a similar change in heart rate in response to stress (Table 3). Non-caffeine consumers had a significant decrease in diastolic blood pressure between rest and stress, compared with caffeine consumers $(-7.20$ vs. $0.24 P=0.0468)$. There

TABLE 1

Patient Characteristics

\begin{tabular}{|c|c|c|c|c|}
\hline Characteristic & Test value & $P$ & Caffeine consumers & Non-caffeine consumers \\
\hline Mean age & $t=1.299$ & 0.1970 & $63.39(12.34)$ & $58.00(17.77)$ \\
\hline$\beta$-blocker use & $\chi^{2}=0.785$ & 0.3757 & $41 \%$ & $27 \%$ \\
\hline Diabetes & $x^{2}=0.323$ & 0.5701 & $37 \%$ & $45 \%$ \\
\hline Sex & $x^{2}=3.404$ & 0.0650 & $57 \% \mathrm{M}$ & $27 \% \mathrm{M}$ \\
\hline Mean body weight (kg) & $t=1.264$ & 0.2093 & $85.30(19.03)$ & 92.99 (19.23) \\
\hline Mean body mass index & $t=2.041$ & 0.0439 & $29.40(6.62)$ & $33.69(6.21)$ \\
\hline Coronary artery disease & $x^{2}=0.068$ & 0.7944 & $31 \%$ & $27 \%$ \\
\hline Hypertension & $\chi^{2}=0.157$ & 0.6916 & $87 \%$ & $91 \%$ \\
\hline Hyperlipidemia & $\chi^{2}=3.562$ & 0.0591 & $62 \%$ & $91 \%$ \\
\hline Smoker & $\chi^{2}=0.764$ & 0.3821 & $20 \%$ & $9 \%$ \\
\hline
\end{tabular}

Data in parentheses are SDs. 
TABLE 2

Symptom Frequency

\begin{tabular}{lccc}
\hline \multicolumn{1}{c}{ Symptom } & $P$ & $\begin{array}{c}\text { Caffeine } \\
\text { consumers }\end{array}$ & $\begin{array}{c}\text { Non-caffeine } \\
\text { consumers }\end{array}$ \\
\hline All symptoms & 0.9579 & & \\
\hline Aminophylline use & 0.0371 & $18 \%$ & $45 \%$ \\
\hline Palpitations & 0.2053 & $2 \%$ & $9 \%$ \\
\hline Shortness of breath & 0.4972 & $23 \%$ & $30 \%$ \\
\hline Flushing & 0.9241 & $10 \%$ & $9 \%$ \\
\hline Chest pain & 0.0013 & $10 \%$ & $45 \%$ \\
\hline Headache & 0.2713 & $14 \%$ & $27 \%$ \\
\hline Nausea or vomiting & 0.8218 & $16 \%$ & $18 \%$ \\
\hline Dizziness & 0.1018 & $20 \%$ & $0 \%$ \\
\hline Feeling hot & 0.5387 & $3 \%$ & $0 \%$ \\
\hline Abdominal pain & 0.2718 & $10 \%$ & $0 \%$ \\
\hline Back pain & 0.7253 & $1 \%$ & $0 \%$ \\
\hline Dysgeusia & 0.2053 & $2 \%$ & $9 \%$ \\
\hline
\end{tabular}

was no difference between caffeine consumers and nonconsumers with regard to change in systolic blood pressure, incidence of hypotension, or rest and stress systolic or diastolic blood pressure. There was no significant difference in the incidence of all-type arrhythmias or atrial fibrillation.

Of the 90 patients who reported caffeine consumption, $50(56 \%)$ reported caffeine intake of less than $200 \mathrm{mg} / \mathrm{d}$, $28(31 \%)$ reported $200-400 \mathrm{mg} / \mathrm{d}$, and $12(13 \%)$ reported more than $400 \mathrm{mg} / \mathrm{d}$. The $\chi^{2}$ and ANOVA analyses for chest pain, administration of aminophylline, and vital signs showed no statistically significant differences as a function of amount of caffeine consumption.

There was no significant difference in the electrocardiographic or nuclear imaging portions of the stress test results between caffeine consumers and non-caffeine consumers.

\section{DISCUSSION}

Our study examined the impact of daily caffeine consumption on symptoms and physiologic changes due to administration of regadenoson for pharmacologic stress testing. There were 3 possible outcomes: first, that daily caffeine consumers would have fewer side effects and a smaller physiologic response than non-caffeine consumers after receiving regadenoson; second, that daily caffeine consumers would experience more frequent side effects and a greater physiologic response to regadenoson; and third, that there would be no difference between consumers and nonconsumers.

The first possible outcome was based on the hypothesis that daily caffeine consumers would have a small amount of residual serum caffeine that would blunt the noncoronary response to regadenoson, since caffeine is an antagonist for the activation of adenosine receptors by regadenoson and the biologic half-life of caffeine (4-6 h) is sufficiently long that some may persist after $12 \mathrm{~h}$ of abstinence (12). Alternatively, daily caffeine intake may cause downregulation of the adenosine receptor signaling cascade, which could result in diminished side effects from adenosine administration. These hypotheses were supported by our data, as daily caffeine consumers had less chest pain and were less likely to receive aminophylline for regadenoson reversal. In addition, daily caffeine consumers exhibited no change in diastolic blood pressure after regadenoson administration, whereas caffeine nonconsumers exhibited the expected small regadenoson-associated decrease in diastolic blood pressure.

The second possible outcome was based on the hypothesis that daily caffeine consumption would potentiate the response to regadenoson through, for example, the upregulation of adenosine receptors, leading to more frequent side effects or a greater physiologic response after regadenoson administration. This hypothesis was not supported by our data.

Our study had several limitations. It was not designed to assess the impact of daily caffeine intake on the accuracy of myocardial perfusion imaging. The effect of timing of caffeine intake on diagnostic accuracy has been previously published by Tejani et al. and Iskandrian et al. $(10,11)$. Although patient

TABLE 3

Mean Physiologic Parameters

\begin{tabular}{|c|c|c|c|}
\hline Parameter & $P$ & Caffeine consumers & Non-caffeine consumers \\
\hline Arrhythmia & 0.8995 & $34 \%$ & $36 \%$ \\
\hline Resting HR (bpm) & 0.0497 & $68.76(12.32)$ & $76.82(15.71)$ \\
\hline Peak HR (bpm) & 0.0314 & 96.52 (16.72) & 108.00 (13.93) \\
\hline HR response (bpm) & 0.4587 & 27.77 (13.80) & 31.18 (18.73) \\
\hline $\mathrm{BP}$ response & 0.3659 & $31 \%$ hyper $/ 22 \%$ hypo & $20 \%$ hyper $/ 10 \%$ hypo \\
\hline Atrial fibrillation & 0.3553 & $3 \%$ & $9 \%$ \\
\hline Hypotension & 0.5387 & $97 \%$ & $91 \%$ \\
\hline Systolic BP response (mm Hg) & 0.7671 & $10.12(23.10)$ & 7.90 (14.37) \\
\hline Diastolic BP response $(\mathrm{mm} \mathrm{Hg})$ & 0.0468 & $0.24(10.46)$ & $-7.20(16.06)$ \\
\hline Rest systolic BP (mm Hg) & 0.4453 & 134.72 (19.89) & 129.70 (17.19) \\
\hline Rest diastolic BP (mm Hg) & 0.7210 & $77.54(13.14)$ & $79.10(11.88)$ \\
\hline Peak systolic BP (mm Hg) & 0.3517 & $144.84(27.28)$ & $136.91(18.82)$ \\
\hline Peak diastolic BP (mm Hg) & 0.2438 & 77.79 (14.72) & 72.27 (14.83) \\
\hline
\end{tabular}

$\mathrm{HR}=$ heart rate; $\mathrm{bpm}=$ beats per minute; $\mathrm{BP}=$ blood pressure; hyper = hypertensive blood pressure response; hypo = hypotensive blood pressure response.

Data in parentheses are SD. 
demographics were relatively similar between caffeine consumers and non-caffeine consumers in our study, the higher mean body mass index and greater percentage of women in the non-caffeine consumers group raise the possibility of confounding. In addition, data on symptoms rely, by necessity, on patient self-reporting. Given the subjectivity of reporting and describing symptoms, one may consider using the significant differences in vital signs as more objective endpoints or using the incidence of aminophylline administration as a defined intervention. Similarly, data on patients' average caffeine intake relied on patient recall. Although questioning about caffeine intake was performed after the stress test to avoid recall bias, the potential for recall bias in retrospective dietary reporting does exist. Additionally, although noncaffeine xanthine derivatives were withheld before the stress test per institutional protocol, we did not specifically evaluate patients for regular use of other xanthine substances, which could pose a potential confounder to our results. Finally, although 101 subjects yielded a sample large enough to achieve statistically significant results, a larger sample size would have been preferable given the small number of non-caffeine users (11 of 101). The percentage of daily caffeine consumers in the general U.S. population is approximately $85 \%$ (13), and our percentage of $89 \%$ daily caffeine consumers is comparable. For future studies, a larger sample size would be required to augment the smaller number of non-caffeine users, which may be obtained by enrolling multiple institutes (although procedural standardization may be more difficult).

\section{CONCLUSION}

Our data show a correlation between caffeine consumption and a lower incidence of chest pain and aminophylline administration during stress with regadenoson, suggesting that caffeine consumption is safe in patients undergoing regadenoson stress and may confer decreased susceptibility to some of the side effects of regadenoson.

\section{DISCLOSURE}

No potential conflict of interest relevant to this article was reported.

\section{REFERENCES}

1. Zoghbi GJ, Iskandrian AE. Selective adenosine agonists and myocardial perfusion imaging. J Nucl Cardiol. 2012;19:126-141.

2. Cerqueira MD. The future of pharmacologic stress: selective A2A adenosine receptor agonists. Am J Cardiol. 2004;94:33D-40D.

3. Londos C, Cooper DMF, Wolff I. Subclasses of external adenosine receptors. Proc Natl Acad Sci USA. 1980;77:2551-2554.

4. van Calker D, Muller M, Hamprecht B. Adenosine regulates via two different types of receptors, the accumulation of cyclic AMP in cultured brain cells. $J$ Neurochem. 1979;33:999-1005.

5. Salvatore CA, Jacobson MA, Taylor HE, Linden J, Johnson RG. Molecular cloning and characterization of the human $\mathrm{A}_{3}$ adenosine receptor. Proc Natl Acad Sci USA. 1993;90:10365-10369.

6. Al Jaroudi W, Iskandrian AE. Regadenoson: a new myocardial stress agent. J Am Coll Cardiol. 2009;54:1123-1130.

7. Hori M, Kitakaze M. Adenosine, the heart, and coronary circulation. Hypertension. 1991;18:565-574.

8. Lexiscan (regadenoson) [package insert]. Northbrook, IL: Astellas Pharma US, Inc.; 2018.

9. Bracco D, Ferrarra JM, Arnaud MJ, Jéquier E, Schutz Y. Effects of caffeine on energy metabolism, heart rate, and methylxanthine metabolism in lean and obese women. Am J Physiol. 1995;269:E671-E678.

10. Tejani FH, Thompson RC, Kristy R, Bukofzer S. Effect of caffeine on SPECT myocardial perfusion imaging during regadenoson pharmacologic stress: a prospective, randomized, multicenter study. Int J Cardiovasc Imaging. 2014;30: 979-989.

11. Iskandrian AE, Bateman TM, Belardinelli L, et al. Adenosine versus regadenoson comparative evaluation in myocardial perfusion imaging: results of the ADVANCE phase 3 multicenter international trial. $J$ Nucl Cardiol. 2007;14:645-658.

12. Benowitz NL. Clinical pharmacology of caffeine. Annu Rev Med. 1990;41:277-288.

13. Mitchell DC, Knight CA, Hockenberry J, Teplansky R, Hartman TJ. Beverage caffeine intakes in the U.S. Food Chem Toxicol. 2014;63:136-142. 